\title{
Numerical solutions of fuzzy integro-differential equations of the second kind
}

\author{
Mohammed S. Bani Issa ${ }^{a}$, Ahmed A. Hamoud ${ }^{\mathrm{b}, *}$, Kirtiwant P. Ghadle \\ ${ }^{a}$ Department of Mathematics, P. E. T. Research Foundation, University of Mysore, Mandya 570401, India. \\ ${ }^{b}$ Department of Mathematics, Taiz University, Taiz, Yemen. \\ ${ }^{c}$ Department of Mathematics, Dr. Babasaheb Ambedkar Marathwada University, Aurangabad, India.
}

\begin{abstract}
In this paper, the Adomian decomposition method, modified Adomian decomposition method, variational iteration method, and homotopy perturbation method are used to solve the fuzzy integro-differential equations. We described the methods and compared the results with their exact solutions to demonstrate the methods' validity and applicability. Examples are provided to illustrate the results.
\end{abstract}

Keywords: Fuzzy integro-differential equations, Adomian decomposition method, modified Adomian decomposition method, variational iteration method, homotopy perturbation method.

2020 MSC: 34A07, 49M27, 65K10, 65H20.

(C)2021 All rights reserved.

\section{Introduction}

The fuzzy differential and integral equations are important part of the fuzzy analysis theory and they have the important value of theory and application in control theory. Later, the area of interest in fuzzy integral equations has been expanded into the fuzzy integro-differential equations (FIDEs). The study of FIDEs forms a suitable setting for mathematical modeling of real-world problems in which uncertainties or vagueness pervade. The topics of FIDEs which is a growing interest for some time, in particular in relation to fuzzy control, have been rapidly developed in recent years [6].

The existence and uniqueness of solutions of FIDEs were investigated by Park and Jeong in [16], Hajighasemi et al. in [7], and Zeinali et al. in [17]. Mikaeilvand et al. in [15] presented the numerical examples of FIDEs using the differential transform method. In [1, 2], Allahviranloo et al. proposed a new technique to solve the FIDEs using definition of generalized differentiability. Hamoud and Ghadle in [11] presented the homotopy analysis method for the first order fuzzy Volterra-Fredholm integro-differential equations.

\footnotetext{
*Corresponding author

Email address: drahmedselwi985@gmail.com (Ahmed A. Hamoud)
}

doi: 10.22436/jmcs.023.01.07 
Motivated by the above work, in this paper we discuss some methods for fuzzy Volterra integrodifferential equations of the form

$$
\tilde{u}^{\prime}(t)=\tilde{f}(t, \tilde{u}(t))+\int_{a}^{t} \tilde{K}(t, s) \tilde{u}(s) d s, \quad t \in J:=(a, b],
$$

with the initial condition

$$
\tilde{\mathrm{u}}(\mathrm{a})=\tilde{\mathrm{u}}_{0},
$$

where function $\tilde{f}: J \times \mathbb{R}_{\mathrm{F}} \longrightarrow \mathbb{R}_{\mathrm{F}}$, crisp function $\tilde{\mathrm{K}}(\mathrm{t}, \mathrm{s})$ are continuous and $\tilde{\mathrm{u}}_{0}$ is a fuzzy number.

\section{Fuzzy Volterra integro-differential equations}

When a physical system is modeled under the differential sense, it finally gives a fuzzy integral equation or a fuzzy integro-differential equation and hence, the solution of fuzzy integro-differential equations have a major role in the fields of science and engineering. Nonlinear fuzzy integro-differential equations are usually hard to solve analytically and exact solutions are scarce. Therefore, they have been of great interest by several authors.

Consider the first order fuzzy integro-differential equations of the form

$$
\tilde{u}^{\prime}(t)=\tilde{f}(t, \tilde{u}(t))+\int_{a}^{t} \tilde{K}(t, s) \tilde{u}(s) d s, \quad t \in(a, b],
$$

with the initial condition

$$
\tilde{\mathrm{u}}(\mathrm{a})=\tilde{\mathrm{u}}_{0} .
$$

The fuzzy integro-differential equations (2.1) is equivalent to the following system

$$
\begin{aligned}
& \underline{u}^{\prime}(t, r)=\underline{f}(t, \underline{u}(t, r))+\int_{a}^{t} \underline{K(t, s) u(s, r)} d s, \\
& \bar{u}^{\prime}(t, r)=\bar{f}(t, \bar{u}(t, r))+\int_{a}^{t} \overline{K(t, s) u(s, r)} d s .
\end{aligned}
$$

We write the parametric form of the given equation (2.1) as follows

$$
\begin{aligned}
& \underline{u}(t, r)=\underline{u}_{0}+\int_{a}^{t} \underline{f}(s, \underline{u}(s, r)) d s+\int_{a}^{t} \int_{a}^{s} \underline{K(t, s) u(s, r)} d r d s, \\
& \bar{u}(t, r)=\bar{u}_{0}+\int_{a}^{t} \bar{f}(s, \bar{u}(s, r)) d s+\int_{a}^{t} \int_{a}^{s} \overline{K(t, s) u(s, r)} d r d s,
\end{aligned}
$$

where $\left[\tilde{u}_{0}\right]_{r}=\left[\underline{u}\left(t_{0}, r\right), \bar{u}\left(t_{0}, r\right)\right], r \in[0,1]$, and

$$
\begin{aligned}
& \underline{K(t, s) u(s, r)}= \begin{cases}K(t, s) \underline{u}(s, r), & K(t, s) \geqslant 0, \\
K(t, s) \overline{\mathcal{u}}(s, r), & K(t, s)<0,\end{cases} \\
& \overline{K(t, s) \mathfrak{u}(s, r)}= \begin{cases}K(t, s) \bar{u}(s, r), & K(t, s) \geqslant 0, \\
K(t, s) \underline{u}(s, r), & K(t, s)<0 .\end{cases}
\end{aligned}
$$

\section{Description of the methods}

We will talk briefly on some dependable techniques for fathoming this sort of equations, where subtleties can be found in $[3,4,8-10,12$ ? -14$]$. 


\subsection{Adomian Decomposition Method (ADM)}

First of all, we discuss Adomian decomposition method. Consider the following fuzzy integrodifferential equations of the form

$$
\begin{aligned}
& \underline{u}(t, r)=\underline{u}_{0}+\int_{a}^{t} \underline{f}(s, \underline{u}(s, r)) d s+\int_{a}^{t} \int_{a}^{s} \underline{K(t, s) u(s, r)} d r d s, \\
& \bar{u}(t, r)=\bar{u}_{0}+\int_{a}^{t} \bar{f}(s, \bar{u}(s, r)) d s+\int_{a}^{t} \int_{a}^{s} \overline{K(t, s) u(s, r)} d r d s .
\end{aligned}
$$

The ADM assume an infinite series solution for the unknowns functions $[\underline{u}, \bar{u}]$, given by

$$
\begin{aligned}
& \underline{u}(t, r)=\sum_{i=0}^{\infty} \underline{u}_{\mathfrak{i}}(t, r), \\
& \bar{u}(t, r)=\sum_{i=0}^{\infty} \bar{u}_{i}(t, r) .
\end{aligned}
$$

The $\tilde{A}_{n}=\left[\underline{A}_{n}, \bar{A}_{n}\right], n \geqslant 0$ are the so-called Adomian polynomial, and $g(t, r)=u_{0}+\int_{a}^{t} f(s, u(s))$ ds we get

$$
\begin{aligned}
& \underline{u}_{0}=\underline{g}(t, r), \\
& \underline{u}_{1}=\int_{a}^{t} \int_{a}^{s} \underline{K(t, s) A_{0}} d r d s, \\
& \quad \vdots \\
& \underline{u}_{n+1}=\int_{a}^{t} \int_{a}^{s} \underline{K(t, s) A_{n}} d r d s, \quad n>0,
\end{aligned}
$$

and

$$
\begin{aligned}
& \overline{\mathrm{u}}_{0}=\bar{g}(\mathrm{t}, \mathrm{r}), \\
& \overline{\mathrm{u}}_{1}=\int_{a}^{t} \int_{a}^{s} \overline{\mathrm{K}(\mathrm{t}, \mathrm{s}) \mathrm{A}_{0}} \mathrm{~d} d s, \\
& \vdots \\
& \overline{\mathrm{u}}_{\mathrm{n}+1}=\int_{a}^{t} \int_{a}^{s} \overline{\mathrm{K}(\mathrm{t}, \mathrm{s}) \mathrm{A}_{\mathrm{n}}} \mathrm{drds}, \quad \mathrm{n}>0 .
\end{aligned}
$$

We approximate $\tilde{u}(t, r)=[\underline{u}(t, r), \bar{u}(t, r)]$ by

$$
\begin{aligned}
\underline{\varphi}_{n} & =\sum_{i=0}^{n-1} \underline{u}_{i}(t, r), \\
\bar{\varphi}_{n} & =\sum_{i=0}^{n-1} \bar{u}_{i}(t, r),
\end{aligned}
$$

where

$$
\lim _{n \rightarrow \infty} \underline{\varphi}_{n}=\underline{u}(t, r), \quad \lim _{n \rightarrow \infty} \bar{\varphi}_{n}=\bar{u}(t, r)
$$




\subsection{Modified Adomian Decomposition Method (MADM)}

In this part, the extended MADM [9] is used to find approximate of nonlinear integro-differential equation (2.1). This method is based on the assumption that the function $g(t, r)$ can be divided into two parts, namely $g_{1}(t, r)$ and $g_{2}(t, r)$. Under this assumption we set

$$
\begin{aligned}
& \underline{u}(t, r)=\underline{g}_{1}(t, r)+\underline{g}_{2}(t, r)+\int_{a}^{t} \int_{a}^{s} \underline{k(t, s) u(s, r)} d r d s, \\
& \bar{u}(t, r)=\bar{g}_{1}(t, r)+\bar{g}_{2}(t, r)+\int_{a}^{t} \int_{a}^{s} \overline{K(t, s) u(s, r)} d r d s .
\end{aligned}
$$

Hence, we can write the following iteration formula

$$
\begin{aligned}
& \underline{\mathrm{u}}_{0}=\underline{\mathrm{g}}_{1}(\mathrm{t}, \mathrm{r}), \\
& \underline{\mathrm{u}}_{1}=\underline{\mathrm{g}}_{2}(\mathrm{t}, \mathrm{r})+\int_{a}^{\mathrm{t}} \int_{a}^{s} \underline{\mathrm{K}(\mathrm{t}, \mathrm{s}) \mathrm{A}_{0}} \mathrm{drds}, \\
& \vdots \\
& \underline{\mathrm{u}}_{n+1}=\int_{a}^{\mathrm{t}} \int_{a}^{s} \underline{\mathrm{K}(\mathrm{t}, \mathrm{s}) \mathrm{A}_{\mathrm{n}}} \mathrm{drds}, \quad \mathrm{n}>0,
\end{aligned}
$$

and

$$
\begin{aligned}
& \overline{\mathrm{u}}_{0}=\bar{g}_{1}(\mathrm{t}, \mathrm{r}), \\
& \overline{\mathrm{u}}_{1}=\bar{g}_{2}(\mathrm{t}, \mathrm{r})+\int_{a}^{\mathrm{t}} \int_{a}^{s} \overline{\mathrm{K}(\mathrm{t}, \mathrm{s}) A_{0}} \mathrm{~d} d s, \\
& \vdots \\
& \overline{\mathrm{u}}_{\mathrm{n}+1}=\int_{a}^{\mathrm{t}} \int_{a}^{s} \overline{\mathrm{K}(\mathrm{t}, \mathrm{s}) A_{\mathrm{n}}} d r d s, \quad n>0 .
\end{aligned}
$$

We approximate $\tilde{u}(t, r)=[\underline{u}(t, r), \bar{u}(t, r)]$ by

$$
\begin{aligned}
\varphi_{n} & =\sum_{i=0}^{n-1} \underline{u}_{i}(t, r), \\
\bar{\varphi}_{n} & =\sum_{i=0}^{n-1} \bar{u}_{i}(t, r),
\end{aligned}
$$

where

$$
\lim _{n \rightarrow \infty} \underline{\varphi}_{n}=\underline{u}(t, r), \quad \lim _{n \rightarrow \infty} \bar{\varphi}_{n}=\bar{u}(t, r) .
$$

\subsection{Variational Iteration Method (VIM)}

Consider the ith equation of (1.1) as a restricted variation, we have the iteration sequence

$$
u_{n+1}=u_{n}+\int_{0}^{x} \lambda\left[u_{n}^{\prime}(s)-\tilde{f}(s, u(s, r))-\int_{a}^{s} K(s, t) u^{\prime}(t) d t\right] d s .
$$

Taking the variation with respect to the independent variable $u_{n}$ and noticing that $\delta u_{n}(0)=0$, we get

$$
\delta u_{n+1}=\delta u_{n}+\left.\lambda(s) \delta u_{n}\right|_{s=x}-\int_{0}^{x} \lambda^{\prime}(s) \delta u_{n} d s=0 .
$$


Then we apply the following stationary conditions

$$
1+\left.\lambda(s)\right|_{s=x}=0,\left.\quad \lambda^{\prime}(s)\right|_{s=x}=0 .
$$

The general Lagrange multiplier, therefore, can be readily identified

$$
\lambda=-1,
$$

and, as a result, we get the following iteration formula

$$
u_{n+1}=u_{n}-\int_{0}^{x}\left[u_{n}^{\prime}(s)-f(s, u(s))-\int_{a}^{s} K(s, t) u^{\prime}(t) d t\right] d s .
$$

Hence, we can write the following iteration formula

$$
\begin{aligned}
& \underline{u}_{n+1}(x, r)=\underline{u}_{n}(x, r)-\int_{0}^{x}\left[\underline{u}_{n}^{\prime}(s, r)-\underline{f}(s, \underline{u}(s, r))-\mu \int_{a}^{s} K(s, t) \underline{u}(t, r) d t\right] d s, \\
& \bar{u}_{n+1}(x, r)=\bar{u}_{n}(x, r)-\int_{0}^{x}\left[\bar{u}_{n}^{\prime}(s, r)-\bar{f}(s, \bar{u}(s, r))-\int_{a}^{s} K(s, t) \bar{u}(t, r) d t\right] d s .
\end{aligned}
$$

\subsection{Homotopy Perturbation Method (HPM)}

Presently we consider the fuzzy integro-differential equations of the second kind in general case $[5,11]$

$$
(1-p)\left[\tilde{u}^{\prime}(t)-\tilde{f}(t)\right]+p\left[\tilde{u}^{\prime}(t)-\tilde{f}(t, \tilde{u}(t))-\int_{a}^{t} K(t, s) \tilde{u}(s) d s\right]=0
$$

Then,

$$
\tilde{u}^{\prime}(t)=\tilde{f}(t, \tilde{u}(t))+p \int_{a}^{t} K(t, s) \tilde{u}(s) d s,
$$

substituting (3.6) in (3.7) and equating the terms with identical powers of $p$, we have

$$
\begin{aligned}
& p^{0}: u_{0}=\underline{g}(t, r), \\
& p^{1}: u_{1}=\int_{a}^{t} \int_{a}^{s} K(t, s) \underline{u}_{0}(s, r) d r d s, \\
& p^{2}: u_{2}=\int_{a}^{t} \int_{a}^{s} K(t, s) \underline{u}_{1}(s, r) d r d s, \\
& p^{3}: u_{3}=\int_{a}^{t} \int_{a}^{s} K(t, s) \underline{u}_{2}(s, r) d r d s,
\end{aligned}
$$

and

$$
\begin{aligned}
& p^{0}: u_{0}=\bar{g}(t, r), \\
& p^{1}: u_{1}=\int_{a}^{t} \int_{a}^{s} K(t, s) \bar{u}_{0}(s, r) d r d s, \\
& p^{2}: u_{2}=\int_{a}^{t} \int_{a}^{s} K(t, s) \bar{u}_{1}(s, r) d r d s, \\
& p^{3}: u_{3}=\int_{a}^{t} \int_{a}^{s} K(t, s) \bar{u}_{2}(s, r) d r d s,
\end{aligned}
$$




\section{Numerical results}

In this section, the comparison is made between ADM, MADM, VIM and HPM for solving fuzzy Volterra integro-differential equations. The efficiency of method is shown in terms of error which is estimated by $\underline{E}(t, r)=\left|\underline{u}_{E}(t, r)-\underline{u}_{A}(t, r)\right|$ and $\bar{E}(t, r)=\left|\bar{u}_{E}(t, r)-\bar{u}_{A}(t, r)\right|$ using MATLAB R2013.

Example 4.1. Consider the following fuzzy Volterra integro-differential equation

$$
\begin{gathered}
u^{\prime}(t, r)=C \frac{1}{12 t}\left(36-5 t^{4}\right)+\int_{0}^{t}\left(t^{2}+s^{2}\right) u(s, r) d s, \\
C=\left[\left(r^{5}+2 r\right) t^{3},\left(6-3 r^{3}\right) t^{3}\right], u(0)=[0,0], 0 \leqslant s \leqslant t \leqslant 1 .
\end{gathered}
$$

The equivalent system is

$$
\begin{aligned}
& \underline{u}^{\prime}(t, r)=\frac{r t^{2}}{12}\left(r^{4}+2\right)\left(36-5 t^{4}\right)+\int_{0}^{t}\left(t^{2}+s^{2}\right) \underline{u}(s, r) d s, \quad \underline{u}(0, r)=0, \\
& \bar{u}^{\prime}(t, r)=\frac{t^{2}}{12}\left(6-3 r^{3}\right)\left(36-5 t^{4}\right)+\int_{0}^{t}\left(t^{2}+s^{2}\right) \bar{u}(s, r) d s, \quad \bar{u}(0, r)=0 .
\end{aligned}
$$

The exact solutions are

$$
\underline{u}(t, r)=\left(r^{5}+2 r\right) t^{3}, \quad \bar{u}(t, r)=\left(6-3 r^{3}\right) t^{3} .
$$

Table 1: Left Bound of Error $\underline{E}(t=0.5, r)$ of the Example 4.1.

\begin{tabular}{lcccc}
\hline $\mathrm{r}$ & $\underline{\mathrm{E}}_{\mathrm{ADM}}$ & $\underline{\mathrm{E}}_{\mathrm{MADM}}$ & $\underline{\mathrm{E}}_{\mathrm{VIM}}$ & $\underline{\mathrm{E}}_{\mathrm{HPM}}$ \\
\hline 0.0 & 0.00000000 & 0.0000000 & 0.0000000 & 0.0000000 \\
0.1 & $7.956810 \mathrm{E}-11$ & $4.823713 \mathrm{E}-10$ & $7.6675 \mathrm{E}-11$ & $2.5125 \mathrm{E}-6$ \\
0.2 & $7.992855 \mathrm{E}-10$ & $9.654662 \mathrm{E}-10$ & $1.5347 \mathrm{E}-10$ & $5.0288 \mathrm{E}-6$ \\
0.3 & $5.998988 \mathrm{E}-10$ & $1.452902 \mathrm{E}-9$ & $2.3095 \mathrm{E}-10$ & $7.5676 \mathrm{E}-6$ \\
0.4 & $5.033500 \mathrm{E}-10$ & $1.954086 \mathrm{E}-9$ & $3.1061 \mathrm{E}-10$ & $1.0178 \mathrm{E}-5$ \\
0.5 & $4.133730 \mathrm{E}-10$ & $2.487103 \mathrm{E}-9$ & $3.9534 \mathrm{E}-10$ & $1.2954 \mathrm{E}-5$ \\
0.6 & $4.360880 \mathrm{E}-10$ & $3.081621 \mathrm{E}-9$ & $4.8984 \mathrm{E}-10$ & $1.6051 \mathrm{E}-5$ \\
0.7 & $4.806100 \mathrm{E}-10$ & $3.781770 \mathrm{E}-9$ & $6.0113 \mathrm{E}-10$ & $1.9698 \mathrm{E}-5$ \\
0.8 & $4.596280 \mathrm{E}-10$ & $4.649055 \mathrm{E}-9$ & $7.3899 \mathrm{E}-10$ & $2.4215 \mathrm{E}-5$ \\
0.9 & $2.190025 \mathrm{E}-9$ & $5.765231 \mathrm{E}-9$ & $9.1641 \mathrm{E}-10$ & $3.0029 \mathrm{E}-5$ \\
1.0 & $2.493445 \mathrm{E}-9$ & $7.235207 \mathrm{E}-9$ & $1.1501 \mathrm{E}-9$ & $3.7686 \mathrm{E}-5$ \\
\hline
\end{tabular}

Table 2: Right Bound of Error $\bar{E}(t=0.5, r)$ of the Example 4.1.

\begin{tabular}{lcccc}
\hline $\mathrm{r}$ & $\overline{\mathrm{E}}_{\text {ADM }}$ & $\overline{\mathrm{E}}_{\text {MADM }}$ & $\overline{\mathrm{E}}_{\mathrm{VIM}}$ & $\overline{\mathrm{E}}_{\mathrm{HPM}}$ \\
\hline 0.0 & $3.986892 \mathrm{E}-9$ & $1.447042 \mathrm{E}-8$ & $2.3001 \mathrm{E}-9$ & $7.5371 \mathrm{E}-5$ \\
0.1 & $3.985401 \mathrm{E}-9$ & $1.446318 \mathrm{E}-8$ & $2.2990 \mathrm{E}-9$ & $7.5333 \mathrm{E}-5$ \\
0.2 & $3.974945 \mathrm{E}-9$ & $1.441254 \mathrm{E}-8$ & $2.2909 \mathrm{E}-9$ & $7.5070 \mathrm{E}-5$ \\
0.3 & $3.946571 \mathrm{E}-9$ & $1.427506 \mathrm{E}-8$ & $2.2691 \mathrm{E}-9$ & $7.4354 \mathrm{E}-5$ \\
0.4 & $2.891310 \mathrm{E}-9$ & $1.400736 \mathrm{E}-8$ & $2.2265 \mathrm{E}-9$ & $7.2959 \mathrm{E}-5$ \\
0.5 & $2.800214 \mathrm{E}-9$ & $1.356601 \mathrm{E}-8$ & $2.1564 \mathrm{E}-9$ & $7.0660 \mathrm{E}-5$ \\
0.6 & $2.664307 \mathrm{E}-9$ & $1.290761 \mathrm{E}-8$ & $2.0517 \mathrm{E}-9$ & $6.7231 \mathrm{E}-5$ \\
0.7 & $2.474641 \mathrm{E}-9$ & $1.198874 \mathrm{E}-8$ & $1.9057 \mathrm{E}-9$ & $6.2444 \mathrm{E}-5$ \\
0.8 & $1.222250 \mathrm{E}-9$ & $1.076599 \mathrm{E}-8$ & $1.7113 \mathrm{E}-9$ & $5.6076 \mathrm{E}-5$ \\
0.9 & $1.898170 \mathrm{E}-9$ & $1.195954 \mathrm{E}-8$ & $1.4617 \mathrm{E}-9$ & $4.7899 \mathrm{E}-5$ \\
1.0 & $1.493445 \mathrm{E}-9$ & $1.235207 \mathrm{E}-8$ & $1.1501 \mathrm{E}-9$ & $3.7686 \mathrm{E}-5$ \\
\hline
\end{tabular}


Example 4.2. Consider the following fuzzy Volterra integro-differential equation

$$
\begin{gathered}
u^{\prime}(t, r)=[2(r-2) \sin (t), 2(2-3 r) \sin (t)]-\int_{0}^{t} u(s, r) d s, \\
u(0)=[3 r-2,2-r], \quad 0 \leqslant s \leqslant t \leqslant 1 .
\end{gathered}
$$

The equivalent system are

$$
\begin{aligned}
& \underline{\mathrm{u}}^{\prime}(\mathrm{t}, \mathrm{r})=2(\mathrm{r}-2) \sin (\mathrm{t})-\int_{0}^{\mathrm{t}} \underline{\mathrm{u}}(\mathrm{s}, \mathrm{r}) \mathrm{ds}, \quad \underline{\mathrm{u}}(0, r)=3 r-2, \\
& \overline{\mathrm{u}}^{\prime}(\mathrm{t}, \mathrm{r})=2(2-3 \mathrm{r}) \sin (\mathrm{t})-\int_{0}^{\mathrm{t}} \overline{\mathrm{u}}(s, r) d s, \quad \overline{\mathrm{u}}(0, r)=2-r .
\end{aligned}
$$

The exact solutions are

$$
\underline{u}(t, r)=(3 r-2)(\cos (t)-t \sin (t)), \quad \bar{u}(t, r)=(2-r)(\cos (t)-t \sin (t)) .
$$

Table 3: Left Bound of Error $\underline{E}(t=0.5, r)$ of the Example 4.2.

\begin{tabular}{lcccc}
\hline $\mathrm{r}$ & $\underline{\mathrm{E}}_{\mathrm{ADM}}$ & $\underline{\mathrm{E}}_{M A D M}$ & $\underline{E}_{\mathrm{VIM}}$ & $\underline{\mathrm{E}}_{\mathrm{HPM}}$ \\
\hline 0.0 & $9.581840 \mathrm{E}-9$ & $9.571280 \mathrm{E}-9$ & $4.694529 \mathrm{E}-8$ & $9.594565 \mathrm{E}-9$ \\
0.1 & $9.262630 \mathrm{E}-9$ & $8.355860 \mathrm{E}-9$ & $4.462684 \mathrm{E}-8$ & $9.484694 \mathrm{E}-9$ \\
0.2 & $8.913970 \mathrm{E}-9$ & $6.894970 \mathrm{E}-9$ & $3.562840 \mathrm{E}-8$ & $9.582860 \mathrm{E}-9$ \\
0.3 & $6.595350 \mathrm{E}-9$ & $5.565460 \mathrm{E}-9$ & $2.171994 \mathrm{E}-8$ & $8.531974 \mathrm{E}-9$ \\
0.4 & $5.236842 \mathrm{E}-9$ & $4.245786 \mathrm{E}-9$ & $2.692641 \mathrm{E}-8$ & $5.584741 \mathrm{E}-9$ \\
0.5 & $4.898290 \mathrm{E}-9$ & $2.794560 \mathrm{E}-9$ & $2.264268 \mathrm{E}-8$ & $2.346386 \mathrm{E}-8$ \\
0.6 & $3.559567 \mathrm{E}-9$ & $1.568732 \mathrm{E}-9$ & $2.348916 \mathrm{E}-8$ & $2.263916 \mathrm{E}-8$ \\
0.7 & $3.218330 \mathrm{E}-10$ & $2.209465 \mathrm{E}-9$ & $1.693848 \mathrm{E}-8$ & $1.186748 \mathrm{E}-8$ \\
0.8 & $2.127786 \mathrm{E}-9$ & $1.722531 \mathrm{E}-9$ & $1.060298 \mathrm{E}-8$ & $1.062748 \mathrm{E}-8$ \\
0.9 & $3.466414 \mathrm{E}-9$ & $2.463523 \mathrm{E}-9$ & $4.726932 \mathrm{E}-8$ & $2.846938 \mathrm{E}-8$ \\
1.0 & $6.768042 \mathrm{E}-9$ & $3.695068 \mathrm{E}-9$ & $4.052464 \mathrm{E}-8$ & $3.463476 \mathrm{E}-8$ \\
\hline
\end{tabular}

Table 4: Right Bound of Error $\overline{\mathrm{E}}(\mathrm{t}=0.5, \mathrm{r})$ of the Example 4.2.

\begin{tabular}{lcccc}
\hline $\mathrm{r}$ & $\overline{\mathrm{E}}_{\text {ADM }}$ & $\overline{\mathrm{E}}_{\text {MADM }}$ & $\overline{\mathrm{E}}_{V I M}$ & $\overline{\mathrm{E}}_{\text {HPM }}$ \\
\hline 0.0 & $7.921801 \mathrm{E}-9$ & $9.691640 \mathrm{E}-9$ & $6.794529 \mathrm{E}-8$ & $9.884469 \mathrm{E}-9$ \\
0.1 & $7.642951 \mathrm{E}-9$ & $9.248750 \mathrm{E}-9$ & $6.868467 \mathrm{E}-8$ & $8.678467 \mathrm{E}-9$ \\
0.2 & $5.891760 \mathrm{E}-9$ & $8.536940 \mathrm{E}-9$ & $6.272415 \mathrm{E}-8$ & $8.432415 \mathrm{E}-9$ \\
0.3 & $5.452860 \mathrm{E}-9$ & $7.759420 \mathrm{E}-9$ & $5.346350 \mathrm{E}-8$ & $7.206382 \mathrm{E}-9$ \\
0.4 & $4.678418 \mathrm{E}-9$ & $7.247950 \mathrm{E}-9$ & $4.790293 \mathrm{E}-8$ & $7.980293 \mathrm{E}-9$ \\
0.5 & $4.899350 \mathrm{E}-9$ & $6.675380 \mathrm{E}-9$ & $4.894225 \mathrm{E}-8$ & $6.754225 \mathrm{E}-8$ \\
0.6 & $4.736930 \mathrm{E}-9$ & $6.127400 \mathrm{E}-9$ & $4.648165 \mathrm{E}-8$ & $6.528165 \mathrm{E}-8$ \\
0.7 & $3.717830 \mathrm{E}-9$ & $5.524870 \mathrm{E}-9$ & $3.412134 \mathrm{E}-8$ & $5.302113 \mathrm{E}-8$ \\
0.8 & $3.574270 \mathrm{E}-9$ & $4.874320 \mathrm{E}-9$ & $3.296047 \mathrm{E}-8$ & $4.178049 \mathrm{E}-8$ \\
0.9 & $3.625673 \mathrm{E}-9$ & $4.484386 \mathrm{E}-9$ & $3.737869 \mathrm{E}-8$ & $4.849686 \mathrm{E}-8$ \\
1.0 & $3.133126 \mathrm{E}-9$ & $3.995720 \mathrm{E}-9$ & $2.853927 \mathrm{E}-8$ & $3.625928 \mathrm{E}-8$ \\
\hline
\end{tabular}

For Problems (4.1) and (4.2), in Tables 1-4, they are observed that the left bound of errors at $t=0.5$ obtained by the fuzzy MADM is competitive with the fuzzy ADM, VIM and HPM. However, for the rest of the errors fuzzy MADM achieved better accuracy compared to fuzzy ADM. Moreover, the errors for the right bound of fuzzy HPM at $t=0.5$ are found to be one decimal place better than the fuzzy VIM. Meanwhile, the results acquired by MADM are almost the same with the results acquired by VIM. In comparison between MADM and HPM, the MADM clearly outperformed the HPM. 


\section{Conclusion}

In the present paper, the ADM, MADM, VIM and HPM were applied to approximate the solution of fuzzy fractional integrodifferential equations. We transformed our problem to system of algebraic equations so that by solving this system we obtained the solution of this kind of equation by considering the type of differentiability. Finally, the solution obtained using the suggested methods show that these approaches can solve the problems effectively.

\section{References}

[1] T. Allahviranloo, S. Abbasbandy, O. Sedaghgatfar, P. Darabi, A new method for solving fuzzy integro-differential equation under generalized differentiability, Neural. Comput. Appl., 21 (2012), 191-196. 1

[2] T. Allahviranloo, M. Khezerloo, O. Sedaghatfar, S. Salahshour, Toward the existence and uniqueness of solutions of second-order fuzzy Volterra integro-differential equations with fuzzy kernel, Neural. Comput. Appl., 22 (2013), 133-141. 1

[3] M. S. Bani Issa, A. A. Hamoud, Some approximate methods for solving system of nonlinear integral equations, Technol. Rep. Kansai Univ., 62 (2020), 388-398. 3

[4] M. S. Bani Issa, A. A. Hamoud, Solving systems of Volterra integro-differential equations by using semi-analytical techniques, Technol. Rep. Kansai Univ., 62 (2020), 685-690. 3

[5] P. R. Bhadane, K. P. Ghadle, A. A. Hamoud, Approximate solution of fractional Black-Schole's European option pricing equation by using ETHPM, Nonlinear Funct. Anal. Appl., 25 (2020), 331-344. 3.4

[6] L. A. Dawood, A. A. Sharif, A. A. Hamoud, Solving higher-order integro differential equations by VIM and MHPM, Int. J. Appl. Math., 33 (2020), 253-264. 1

[7] S. Hajighasemi, T. Allahviranloo, M. Khezerloo, M. Khorasany, S. Salahshour, Existence and uniqueness of solutions of fuzzy Volterra integro-differential equations, 13th International Conference (Dortmund, Germany), 2010 (2010), 491-500. 1

[8] A. A. Hamoud, K. P. Ghadle, Existence and uniqueness of solutions for fractional mixed Volterra-Fredholm integrodifferential equations, Indian J. Math., 60 (2018), 375-395. 3

[9] A. A. Hamoud, K. P. Ghadle, The approximate solutions of fractional Volterra-Fredholm integro-differential equations by using analytical techniques, Probl. Anal. Issues Anal., 7 (2018), 41-58. 3.2

[10] A. A. Hamoud, K. P. Ghadle, Existence and uniqueness of the solution for Volterra-Fredholm integro-differential equations, J. Sib. Fed. Univ. Math. Phys., 11 (2018), 692-701. 3

[11] A. A. Hamoud, K. P. Ghadle, Homotopy analysis method for the first order fuzzy Volterra-Fredholm integro-differential equations, Indones. J. Electr. Eng. Comput. Sci., 11 (2018), 857-867. 1, 3.4

[12] A. A. Hamoud, K. P. Ghadle, Usage of the homotopy analysis method for solving fractional Volterra-Fredholm integrodifferential equation of the second kind, Tamkang J. Math., 49 (2018), 301-315. 3

[13] A. A. Hamoud, K. P. Ghadle, Some new existence, uniqueness and convergence results for fractional Volterra-Fredholm integro-differential equations, J. Appl. Comput. Mech., 5 (2019), 58-69.

[14] K. H. Hussain, A. H. Hamoud, N. M. Mohammed, Some new uniqueness results for fractional integro-differential equations, Nonlinear Funct. Anal. Appl., 24 (2019), 827-836. 3

[15] N. Mikaeilvand, S. Khakrangin, T. Allahviranloo, Solving fuzzy Volterra integro-differential equation by fuzzy differential transform method, Proceedings of the 7th Conference of the European Society for Fuzzy Logic and Technology (Aix-Les-Bains, France), 2011 (2011), 891-896. 1

[16] J. Y. Park, J. U. Jeong, On existence and uniqueness of solutions of fuzzy integro differential equations, Indian J. Pure Appl. Math., 34 (2003), 1503-1512. 1

[17] M. Zeinali, S. Shahmorad, K. Mirnia, Fuzzy integro-differential equations: Discrete solution and error estimation, Iran. J. Fuzzy Syst., 10 (2013), 107-122. 1 